\title{
Rotor parameter determination for the Brushless Doubly Fed (Induction) Machine (BDFM)
}

\author{
R. A. McMahon*, P. C. Roberts ${ }^{\dagger}$, M. R. Tatlow*, E. Abdi ${ }^{\ddagger}$, A. Broekhof* and S. Abdi* \\ * Electrical Engineering Division, University of Cambridge, 9 JJ Thomson Avenue, Cambridge, CB3 0FA, UK \\ $\{$ ram1,mrt39,ab940,sa612\}@cam.ac.uk \\ $\dagger$ Cambridge Medical Robotics Ltd., Crome Lea Business Park, Madingley Road, Cambridge, CB23 7PH, UK \\ paul.roberts@cmedrobotics.com \\ ‡ Wind Technologies Ltd., Cambridge Science Park, Cambridge, CB4 OEY, UK \\ ehsan.abdi@windtechnologies.com
}

\begin{abstract}
A procedure has been established for obtaining equivalent circuit parameters for the Brushless Doubly Fed Machine (BDFM), also known as the Brushless Doubly Fed Induction Machine (DFIM), by transforming a reduced coupled-circuit model into a sequence component form. This approach takes advantage of the model reduction procedure's ability to reduce a complex rotor structure to a single equivalent loop without significant loss of accuracy. An alternative method based on winding factors has also been developed and includes coupling via harmonic fields. Parameters determined in both ways are in close agreement with those determined from Finite Element Analysis (FEA) and experimentally. The advantages of each method is discussed in the conclusion section.
\end{abstract}

\begin{tabular}{ll} 
& \multicolumn{1}{c}{ LIST OF SYMBOLS } \\
$X_{1}, X_{2}, X_{r}$ & indicating a stator 1, 2, or rotor quantity $X$ \\
$p_{1}, p_{2}$ & stator winding pole pairs (principal fields) \\
$g$ & effective air-gap \\
$l, d$ & stack length, mean air-gap diameter \\
$w_{s}$ & slot mouth opening \\
$\omega_{r}$ & rotor angular velocity \\
$\mathbb{R}, \mathbb{C}, \mathbb{N}, \mathbb{Z}$ & fields of real and complex numbers, sets of natural numbers and integers \\
$\operatorname{diag}\left(X_{1}, X_{2}, \cdots\right)$ & Diagonal matrix with scalars or matrices $X_{1}, X_{2}$ etc. \\
$k_{w}$ & winding factor for a single loop in a nest \\
$N_{e f f}$ & effective turns \\
$Z_{e f f}$ & Impedance of individual rotor loop $(\Omega)$ \\
$n_{r}$ & rotor turns ratio
\end{tabular}

\section{INTRODUCTION}

Contemporary interest in the BDFM principally arises as it is a potential alternative to the slip-ring induction generator widely used in wind turbines although it can also be used as a drive. In recent years several large prototype machines have been reported [1]-[3]. The BDFM has two windings on a common stator core, configured for different, non-coupling pole numbers ( $p_{1}$ and $p_{2}$ associated with stator 1 and stator 2 respectively). A special design of rotor cross-couples between the two stator windings. One stator winding is connected directly to the grid; the other is supplied with variable voltage and frequency through a converter. The machine is normally run in a synchronous mode, with an appropriate controller, in which the shaft speed is set by the frequencies supplied to the stator windings. In this mode, the BDFM operates in a similar way to the doubly-fed induction generator with the torque related to the load angle and a grid side power factor which can be varied by adjusting the control winding voltage.

The presence of two stators means that there are more variables to consider than in a single winding machine and the determination of the ratings of the second stator and associated inverter is complicated by the fact that control of torque and power factor are not fully decoupled. The machine's operating conditions can be directly calculated using the method described by Williamson et. al [4], the coupled circuit method [5] or by Finite Element Analysis (FEA) [6]. As an alternative, the equivalent circuit approach provides an effective way of determining operating conditions but relies on having accurate machine parameters. In addition, the equivalent circuit forms a valuable design tool, enabling candidate machine designs to be rapidly evaluated. In particular rotor parameters are important in determining the pull out torque of the machine as well as low-voltage ride through performance in wind turbine applications. Some parameters can be found by standard methods but the calculation of parameters for the usual type of rotor with nests of multiple loops is not straightforward [7]. In addition, they are are speed dependent. 
Two methods of obtaining rotor parameters are given in this paper and applied to the nested loop type of rotor with $p_{1}+p_{2}$ equally spaced sets of $N$ nested loops, as shown in Figure 1. One approach is based on the model reduction procedure starting from a coupled-circuit model which leads to a single set of $d q$ parameters for the rotor, independent of operating speed [5]. Implicit in this is that stator to rotor couplings are assumed to take place only via the principal $\left(p_{1}+p_{2}\right)$ fields but couplings between rotor loops via harmonic fields are included. The $d q$ components are then transformed to give the rotor parameters.

The alternative method is an extension of the approach based on winding factors given in [7] to include coupling via harmonic fields. The values obtained from the two models are compared to those found experimentally and by FEA.

\section{A. Equivalent Circuit}

The operation of the BDFM can be described by a per-phase equivalent circuit of the form show in Figure 2a with the parameters summarized in Table I. Parameters are referred to the power winding and iron losses are neglected. The ', '

TABLE I: Equivalent circuit parameters

\begin{tabular}{ll}
\hline Parameter & Description \\
\hline$R_{1}, R_{2}$ & Stator 1 and 2 resistances \\
$R_{r}$ & Rotor resistance \\
$L_{m 1}, L_{m 2}$ & Stator 1 and 2 magnetizing inductances \\
$L_{1}, L_{2}$ & Stator 1 and 2 leakage inductances \\
$L_{r}$ & Rotor inductance \\
\hline
\end{tabular}

modifier denotes that the quantity is referred. Slips $s_{1}$ and $s_{2}$ are given by (11) and (12). The leakage inductances cannot be directly measured so the simplified equivalent circuit [8] shown in Figure $2 b$ is used. In the simplified equivalent circuit, $L_{1}$ and $L_{2}$ have been absorbed into $L_{m 1,2}$ and as a result the values of the magnetizing and rotor leakage inductances will change. There will also be a modification to the turns ratio, $n_{r}$, and hence all referred parameters.

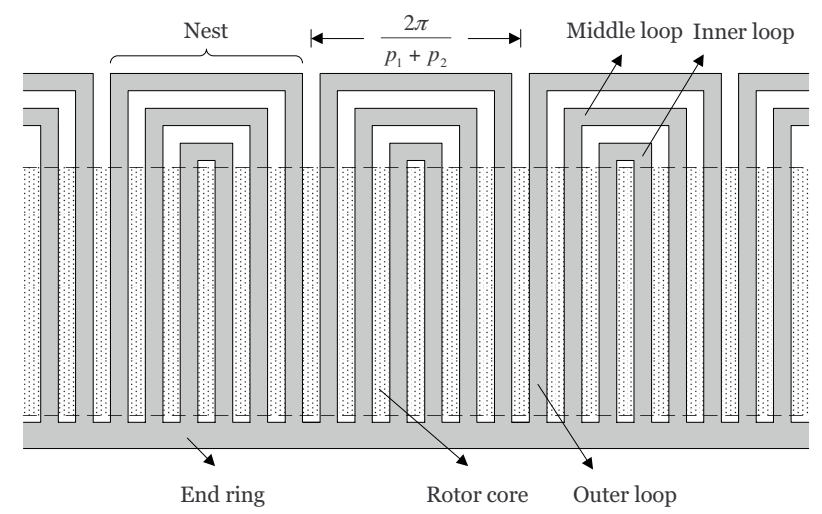

Fig. 1: Nested loop rotor design, with three loops per nest

\section{THE COUPLED-CIRCUIT METHOD}

\section{A. Steady-state analysis by complex decomposition}

The coupled-circuit model relating the stator and rotor voltages and currents has been presented in [5]. It was shown that the model can be reduced so that the rotor-rotor and stator-rotor couplings are dependent on a single $d q$ pair, each. This reduction

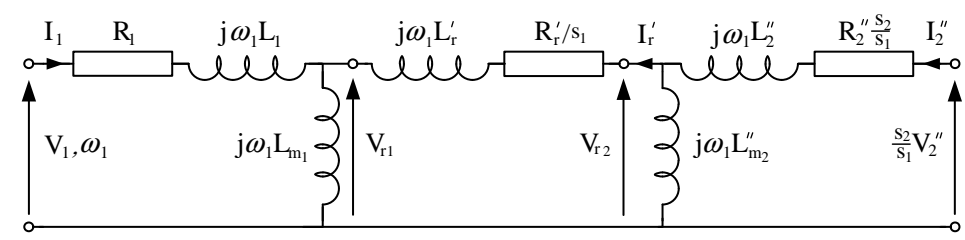

(a) Full per-phase equivalent circuit

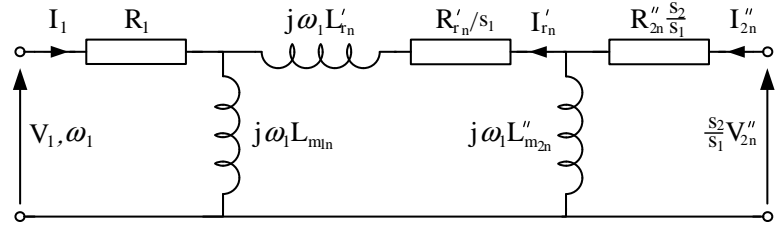

(b) Reduced per-phase equivalent circuit

Fig. 2: Per-phase BDFM equivalent circuit referred to the power winding 
is equivalent to modelling the rotor as having a single loop per nest. The model can then be written as:

$$
\left[\begin{array}{c}
v_{s} \\
0
\end{array}\right]=\left[\begin{array}{cc}
M_{s} & M_{s r} \\
M_{s r}^{\mathrm{T}} & M_{r}
\end{array}\right] \frac{d}{d t}\left[\begin{array}{l}
i_{s} \\
i_{r}
\end{array}\right]+\left(\left[\begin{array}{cc}
R_{s} & 0 \\
0 & R_{r_{1}}
\end{array}\right]+\omega_{r}\left[\begin{array}{cc}
Q_{s} & Q_{s r} \\
0 & 0
\end{array}\right]\right)\left[\begin{array}{l}
i_{s} \\
i_{r}
\end{array}\right]
$$

A transformation into a complex form, as first undertaken by Li et al. for the case of a BDFM with a nested loop rotor [9], ultimately allows parameters to be found for an equivalent circuit. The $d q 0$ current components may be transformed into two counter-rotating vectors (plus a zero component) using the following relationship:

$$
\begin{gathered}
{\left[\begin{array}{l}
i^{+} \\
i^{-} \\
i_{0}
\end{array}\right]=\left[\begin{array}{cr}
{\left[\begin{array}{c}
\mathcal{T}_{c}^{+} \\
\mathcal{T}_{c}^{-}
\end{array}\right]} & 0 \\
0 & 1
\end{array}\right]\left[\begin{array}{l}
i_{d} \\
i_{q} \\
i_{0}
\end{array}\right]} \\
\text { where } \mathcal{T}_{c}^{+}=\frac{1}{\sqrt{2}}\left[\begin{array}{ll}
1 & j
\end{array}\right], \mathcal{T}_{c}^{-}=\frac{1}{\sqrt{2}}\left[\begin{array}{ll}
1 & -j
\end{array}\right]
\end{gathered}
$$

where superscripts + and - represent the forward and backward rotating vectors. This transform assigns the $d$ and $q$ axes to the real and imaginary axes, respectively. Along with ensuring a unitary transform, the counter-rotating vector is used for the stator 2, whose rotating magnetic field appears anti-phase in the rotor reference frame. The transformation matrix for the reduced-order model will consist of three copies of $\mathcal{T}_{c}^{+}$and $\mathcal{T}_{c}^{-}$and two 1s along the diagonal. Although, from a mathematical point of view, these elements comprising the transformation matrix may be put in any order, the whole transformation matrix for the reduced-order model can be chosen to be:

$$
\mathcal{T}_{\text {cmplx }}=\left[\begin{array}{ccccc}
\mathcal{T}_{c}^{+} & 0 & 0 & 0 & 0 \\
0 & 0 & \mathcal{T}_{c}^{-} & 0 & 0 \\
0 & 0 & 0 & 0 & \mathcal{T}_{c}^{+} \\
\mathcal{T}_{c}^{-} & 0 & 0 & 0 & 0 \\
0 & 0 & \mathcal{T}_{c}^{+} & 0 & 0 \\
0 & 0 & 0 & 0 & \mathcal{T}_{c}^{-} \\
0 & 1 & 0 & 0 & 0 \\
0 & 0 & 0 & 1 & 0
\end{array}\right]
$$

The matrix has been organized to group forward rotating, backward rotating and zero current components together. The two final 1s are for the stator zero components. As $\mathcal{T}_{\text {cmplx }}$ is unitary, the currents can be transformed as follows:

$$
\left[\begin{array}{c}
i_{c_{s 1}}^{+} \\
i_{c_{s 2}}^{-} \\
i_{c_{r}}^{+} \\
i_{c_{s 1}}^{-} \\
i_{c_{s 2}}^{+} \\
i_{c_{r}}^{-} \\
i_{c_{\emptyset}}^{-}
\end{array}\right]=\mathcal{T}_{\text {cmplx }}\left[\begin{array}{c}
i_{d q_{s 1}} \\
i_{d q_{s 2}} \\
i_{d q_{r}}
\end{array}\right] \Rightarrow\left[\begin{array}{c}
i_{d q_{s 1}} \\
i_{d q_{s 2}} \\
i_{d q_{r}}
\end{array}\right]=\mathcal{T}_{\text {cmplx }}^{*}\left[\begin{array}{c}
i_{c_{s 1}}^{+} \\
i_{c_{s 2}}^{-} \\
i_{c_{r}}^{+} \\
i_{c_{s 1}}^{-} \\
i_{c_{s 2}}^{+} \\
i_{c_{r}}^{-} \\
i_{c_{\emptyset}}^{+}
\end{array}\right]
$$

where subscript $c$ indicates that the quantity has been transformed to the complex form. The voltages can be transformed similarly. These vectors can then be condensed by defining:

$$
\left.\left.\left[\begin{array}{c}
i_{c_{s 1}}^{+} \\
i_{c_{s 2}}^{-} \\
i_{c_{r}}^{+} \\
i_{c_{s 1}}^{-} \\
i_{c_{s 2}}^{+} \\
i_{c_{r}}^{-} \\
i_{c_{\emptyset}}
\end{array}\right]\right]:=\left[\begin{array}{c}
i_{c}^{+} \\
i_{c}^{-} \\
i_{c_{\emptyset}}^{-}
\end{array}\right],\left[\begin{array}{c}
v_{c_{s 1}}^{+} \\
v_{c_{s 2}}^{-} \\
v_{c_{r}}^{+} \\
v_{c_{s 1}}^{-} \\
v_{c_{s 2}}^{+} \\
v_{c_{r}}^{-} \\
v_{c_{\emptyset}}
\end{array}\right]\right]:=\left[\begin{array}{c}
v_{c}^{+} \\
v_{c}^{-} \\
v_{c_{\emptyset}}^{+}
\end{array}\right]
$$

This transformation is applied to the reduced-order BDFM $d q$ model given in equation (1) by multiplying by $\mathcal{T}_{\text {cmplx }}$ from the left and by $\mathcal{T}_{\text {cmplx }}^{*}$ from the right. Substituting for the complex voltages and currents using equation (5) gives a model of the form:

$$
\left[\begin{array}{c}
v_{c}^{+} \\
v_{c}^{-} \\
v_{c_{\emptyset}}
\end{array}\right]=\left(R_{c}+\omega_{r} Q_{c}\right)\left[\begin{array}{l}
i_{c}^{+} \\
i_{c}^{-} \\
i_{c_{\emptyset}}
\end{array}\right]+M_{c} \frac{d}{d t}\left[\begin{array}{l}
i_{c}^{+} \\
i_{c}^{-} \\
i_{c_{\emptyset}}
\end{array}\right]
$$

Details for $R_{c}, Q_{c}$ and $M_{c}$ are given in appendix A. 


$$
\begin{array}{lc}
\text { Stator 1 } & \text { Complex form voltages } \\
\text { Voltage: } & {\left[\begin{array}{l}
v_{c_{s 1}}^{+} \\
v_{c_{s 1}}^{-} \\
v_{c_{\emptyset_{1}}}^{-}
\end{array}\right]=\left[\begin{array}{c}
\sqrt{\frac{3}{2}}\left|V_{1}\right| \exp \left(j\left(p_{1} \theta_{r}-\omega_{1} t-\alpha_{1}\right)\right) \\
\sqrt{\frac{3}{2}}\left|V_{1}\right| \exp \left(-j\left(p_{1} \theta_{r}-\omega_{1} t-\alpha_{1}\right)\right) \\
0
\end{array}\right]} \\
\text { Stator 2 } & {\left[\begin{array}{l}
v_{c_{s 2}}^{+} \\
v_{s_{s 2}}^{-} \\
v_{c_{\emptyset_{2}}}
\end{array}\right]=\left[\begin{array}{c}
\sqrt{\frac{3}{2}}\left|V_{2}\right| \exp \left(j\left(p_{2} \theta_{r}-\int \omega_{2} d t-\alpha_{2}\right)\right) \\
\sqrt{\frac{3}{2}}\left|V_{2}\right| \exp \left(-j\left(p_{2} \theta_{r}-\int \omega_{2} d t-\alpha_{2}\right)\right) \\
0
\end{array}\right]}
\end{array}
$$

TABLE II: Complex stator voltages. $\left|V_{1}\right|$ and $\left|V_{2}\right|$ are rms magnitudes and $\alpha_{1}$ and $\alpha_{2}$ are phase offsets

The structure of (7) shows that the equations relating $i_{c}^{+}$to $v_{c}^{+}, i_{c}^{-}$to $v_{c}^{-}$, and $i_{c_{\emptyset}}$ to $v_{c_{\emptyset}}$ are all independent of one another. Furthermore, it is easy to see that due to the structure of equation (7) if $v_{c}^{-}=\bar{v}_{c}^{+}$then $i_{c}^{-}=\bar{i}_{c}^{+}$, where an overbar denotes the complex conjugate, as shown in table II. Therefore, in balanced three phase conditions, the BDFM may be fully described using the forward-rotating components only:

$$
v_{c}^{+}=\left(R_{c}^{+}+\omega_{r} Q_{c}^{+}\right) i_{c}^{+}+M_{c}^{+} \frac{d i_{c}^{+}}{d t}
$$

\section{B. Equivalent circuit representation}

In the steady-state, an equivalent circuit representation of equation (8) can be derived. With the machine operating in synchronous mode, i.e.

$$
\omega_{r}=\frac{\omega_{1}+\omega_{2}}{p_{1}+p_{2}}
$$

the frequency of the currents in the rotor reference frame are given by $\omega_{\text {sync }}=p_{1} \omega_{r}-\omega_{1}=-p_{2} \omega_{r}+\omega_{2}$ [5]. Therefore, equation (8) becomes:

$$
\sqrt{\frac{3}{2}} V_{c}=\left(R_{c}^{+}+\omega_{r} Q_{c}^{+}+j \omega_{\text {sync }} M_{c}^{+}\right) \sqrt{\frac{3}{2}} I_{c}
$$

where $\sqrt{\frac{3}{2}} V_{c}$ and $\sqrt{\frac{3}{2}} I_{c}\left(V_{c}, I_{c} \in \mathbb{C}^{3}\right)$ are vectors of appropriate steady-state components of current and voltage; $\omega_{r} Q_{c}^{+}+$ $j \omega_{\text {sync }} M_{c}^{+}$is given in appendix A by equation (29).

Following [10], slips between stators 1 and 2 and the rotor are defined as follows:

$$
\begin{aligned}
& s_{1} \triangleq \frac{\omega_{1} / p_{1}-\omega_{r}}{\omega_{1} / p_{1}}=\frac{\omega_{1}-p_{1} \omega_{r}}{\omega_{1}}=-\frac{\omega_{\text {sync }}}{\omega_{1}} \\
& s_{2} \triangleq \frac{\omega_{2} / p_{2}-\omega_{r}}{\omega_{2} / p_{2}}=\frac{\omega_{2}-p_{2} \omega_{r}}{\omega_{2}}=\frac{\omega_{\text {sync }}}{\omega_{2}}
\end{aligned}
$$

Substituting (11), (12) and (29) into (10) gives (subject to $\omega_{1} \neq 0$ and $\omega_{\text {sync }} \neq 0$ ):

$$
\left[\begin{array}{c}
V_{1} \\
\frac{s_{2}}{s_{1}} V_{2} \\
0
\end{array}\right]=\left(-j \omega_{1}\left[\begin{array}{ccc}
L_{c_{s 1}} & 0 & M_{c_{s 1 r}} \\
0 & L_{c_{s 2}} & M_{c_{s 2 r}} \\
M_{c_{s 1 r}}^{*} & M_{c_{s 2 r}}^{*} & M_{c_{r}}
\end{array}\right]+\left[\begin{array}{ccc}
R_{1} & 0 & 0 \\
0 & \frac{s_{2}}{s_{1}} R_{2} & 0 \\
0 & 0 & \frac{1}{s_{1}} R_{r}
\end{array}\right]\right)\left[\begin{array}{c}
I_{1} \\
I_{2} \\
I_{r}
\end{array}\right]
$$

where $L_{c_{s 1}}=L_{1}-M_{1}$ and $L_{c_{s 2}}=L_{2}-M_{2}$. Furthermore, the scaling and frequency dependent terms of (10) cancel out, yielding complex phasor sets $\left[\begin{array}{c}V_{1} \\ V_{2} \\ 0\end{array}\right]$ and $\left[\begin{array}{l}I_{1} \\ I_{2} \\ I_{r}\end{array}\right]$ with physical quantities.

Equation (13) is in a form which admits an equivalent circuit representation. If the rotor has (or is modelled to have) only one set of coils (i.e. $N=1$ ) then any phase shift will be eliminated by an appropriate rotation matrix, $\mathcal{T}_{\text {rot }}$, and hence $M_{c_{r}}$, $M_{c_{s 1 r}}$ and $M_{c_{s 2 r}}$ become purely real.

In this case, a useful equivalent circuit representation may be derived based on equation (13), as shown in figure 3 . This form of the equivalent circuit is the same as that found in [9], [11].

To obtain the equivalent circuit depicted in fig. $2 \mathrm{a}$, it is necessary to split the inductance terms into their constituent parts:

$$
\begin{aligned}
& L_{c_{s 1}}=L_{1_{f}}+L_{1_{h}}+L_{1_{l}} \\
& L_{c_{s 2}}=L_{2_{f}}+L_{2_{h}}+L_{2_{l}} \\
& M_{c_{r}}=L_{r_{1}}+L_{r_{2}}+L_{r_{h}}+L_{r_{l}}
\end{aligned}
$$




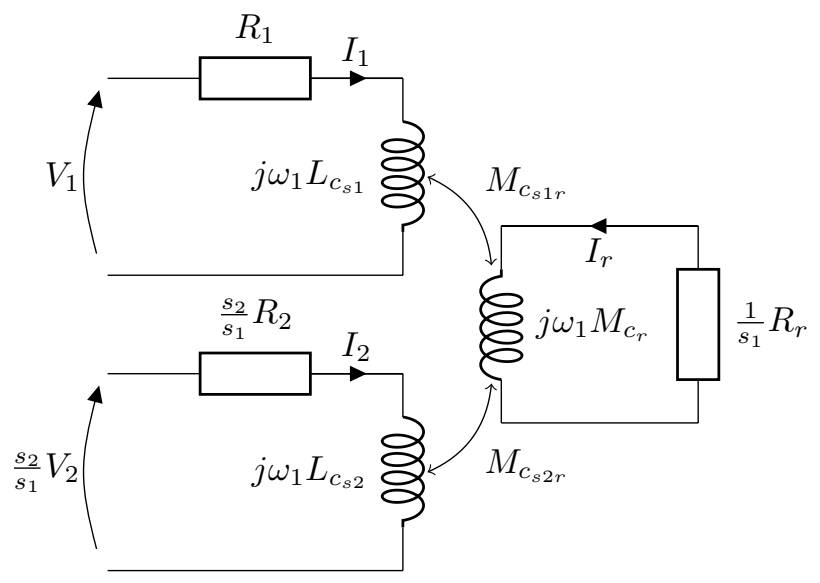

Fig. 3: Coupled coils per-phase equivalent circuit for a single rotor circuit BDFM

$L_{1_{f}}$ and $L_{2_{f}}$ represent the self-inductance of the stator windings arising from the principal fields $\left(p_{1}\right.$ and $\left.p_{2}\right)$ and the $L_{r_{1}}$ and $L_{r_{2}}$ terms represent the rotor self-inductances linking the rotor to $p_{1}$ and $p_{2}$ respectively. The inductance due to all remaining harmonics are grouped under the subscript $h$ terms. Inductance due to leakage is self-inductance arising from flux which does not cross the air-gap, and is represented by subscript $l$ terms.

The individual harmonic terms can be obtained by calculating the machine parameters of equation (13) from a Fourier series representation [5]. For each harmonic a separate inductance matrix will be given. As the $d q 0$ transformation and model reduction procedure given in [5], and complex form transformation described above, are linear, they may be applied directly to each harmonic component individually (note that the matrix used in the model reduction for each harmonic will always be the one derived for the full system).

Using the relationships shown in equation (14) figure 3 can be converted to figure 4.

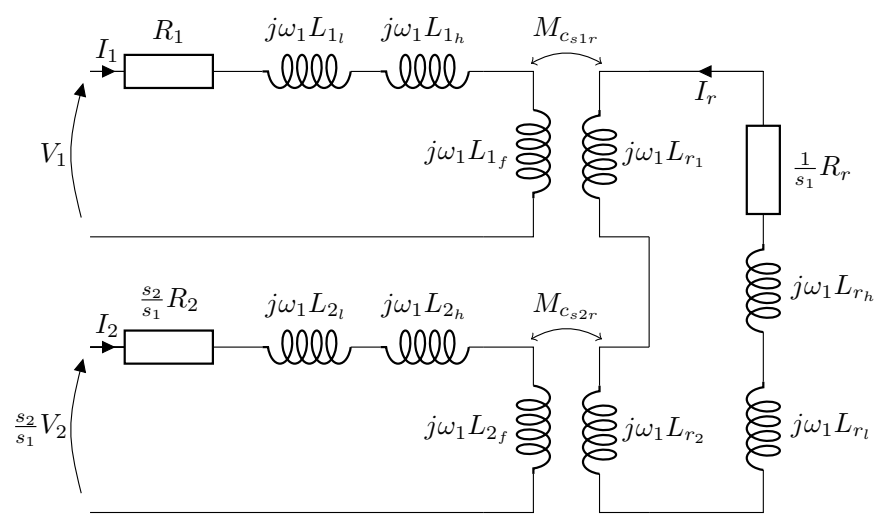

Fig. 4: Coupled coils Equivalent Circuit Separating $M_{c_{r}}, L_{c_{s 1}}, L_{c_{s 2}}$

Figure 4 may be further transformed into a ' $T$ ' equivalent form with transformers [12]. It is convenient to choose the turns ratio in each case so that it is the effective turns ratio for each coupling. The effective turns ratio is the square root of the ratio of the fundamental space harmonic self-inductance terms for the rotor and the stator [13, p. 381]. Therefore the effective turns ratios for stator 1-rotor and stator 2-rotor are:

$$
N_{1}=\sqrt{\frac{L_{1_{f}}}{L_{r_{1}}}}, \quad N_{2}=\sqrt{\frac{L_{2_{f}}}{L_{r_{2}}}}
$$

Because $L_{1_{f}}, L_{r_{1}}$ and $L_{2_{f}}, L_{r_{2}}$ represent couplings between same pole number fields with no parasitic effects, the coupling in each case is perfect, so:

$$
\left|M_{c_{s 1 r}}\right|=\sqrt{L_{1_{f}} L_{r_{1}}}, \quad\left|M_{c_{s 2 r}}\right|=\sqrt{L_{2_{f}} L_{r_{2}}}
$$

Then, converting the coupled coils arrangements of figure 4 gives the equivalent circuit as shown in figure 5 . 


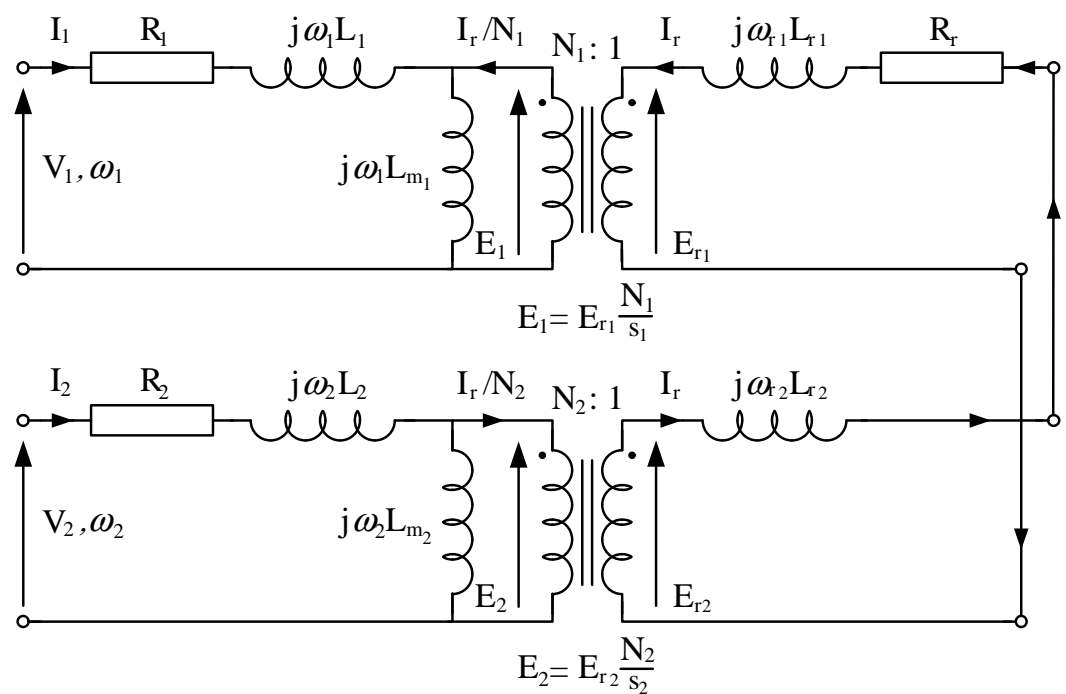

Fig. 5: BDFM Equivalent circuit with separate inductance components

Figure 5 can be easily converted to figure 2 a by noting that, from (11) and (12), $\omega_{\text {sync }}=-s_{1} \omega_{1}$. The circuit of figure 5 may be recognized as two standard induction machine equivalent circuits with rotors connected together. Furthermore, figure 5 may be referred to stator 1 or 2 as in [8]. This equivalent circuit expresses the parameters in table I and is therefore the endpoint of the coupled-circuit parameter-determination method.

\section{WINDING FACTOR METHOD}

\section{A. Rotor model}

The winding factor method of analysis centres on the loops of the nested loop rotor which are effectively in parallel and have mutual couplings via the principal fields and their space harmonics. Each loop has winding factors for the principal fields, magnetizing inductances for each space harmonic, emfs produced by mutual inductances for the various space harmonics in response to the flow of currents in other rotor loops and via coupling to the stator. In addition, each loop has a leakage reactance which can be estimated by conventional means and a resistance which can be calculated or measured. These are shown in Figure 6. In addition to the rotor loops, fictitious stator coils, with one effective turn, for the two principal fields are shown for convenience of analysis.

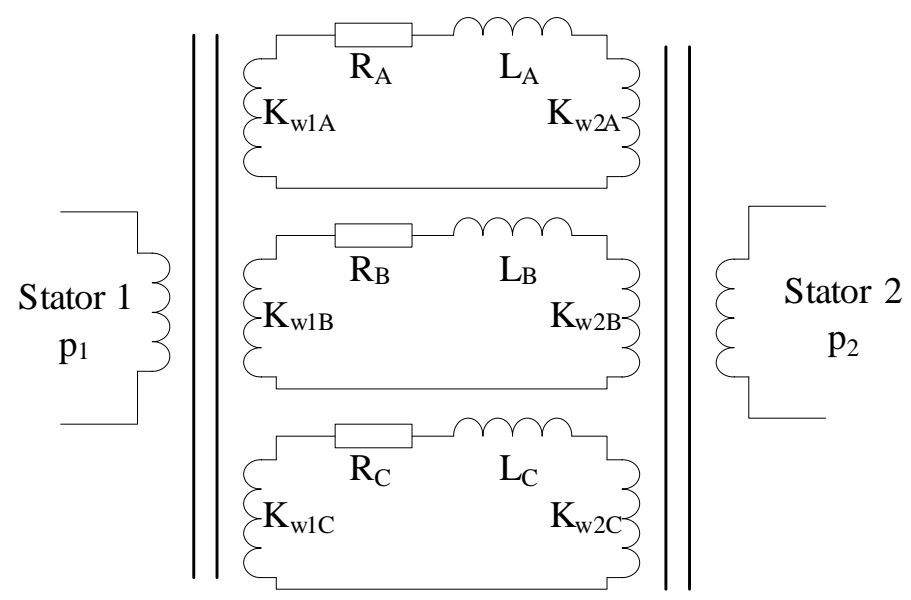

Fig. 6: Nested loop electrical circuit, with three loops per nest

Space harmonics of the principal fields manifest themselves as magnetizing and mutual inductances in each loop. The space harmonics, $p$ that exist (harmonic order $n$ ) are described in [7] as: 


$$
p \in\left\{\left\{p_{1}+m\left(p_{1}+p_{2}\right)\right\} \cup\left\{p_{2}+m\left(p_{1}+p_{2}\right)\right\}\right\}, m \in \mathbb{N}
$$

The winding factor, $k_{w}$, for a single loop in a nest, as shown in Figure 6 is:

$$
k_{w}=\sin \left(\beta \frac{p}{2}\right) \frac{\sin \left(\frac{\omega_{s r} p}{2}\right)}{\frac{\omega_{s r} p}{2}}
$$

Where $\beta$ is the individual coil span of the loop, $p$ is the rotor harmonic pole pair given in the set (17) and $\omega_{s r}$ is the angular slot mouth span.

The self-inductance evaluated for all rotor loops of a specific span, taking into account each harmonic pole-pair is calculated as:

$$
\left[\begin{array}{ccc}
L_{m_{1,1}} & \ldots & L_{m_{1, n}} \\
\vdots & \ddots & \vdots \\
L_{m_{i, 1}} & \ldots & L_{m_{i, n}}
\end{array}\right]=\left(\frac{\mu_{0} l d q}{\pi g}\right)\left[\begin{array}{ccc}
\left(\frac{N_{e f f_{1,1}}}{p_{1}}\right)^{2} & \ldots & \left(\frac{N_{e f f_{1, n}}}{p_{1}}\right)^{2} \\
\vdots & \ddots & \vdots \\
\left(\frac{N_{e f f_{i, 1}}}{p_{i}}\right)^{2} & \ldots & \left(\frac{N_{e f f_{i, n}}}{p_{i}}\right)^{2}
\end{array}\right]
$$

Where $q$ is the summation of the pole-pairs $\left(p_{1}+p_{2}\right), n$ is the loop number (A, B, C etc), $N_{\text {eff }}$ is the effective rotor turns found for the $i^{t h}$ harmonic being analyzed, the first two components being the principal fields (i.e $i=1$ corresponds to the first harmonic pole pair in the set $p$ ).

The total magnetizing inductance for each loop from the rotor space harmonics (excluding the two principal fields) is calculated as:

$$
\left[\begin{array}{c}
L_{m_{l o o p, 1}} \\
L_{m_{l o o p, 2}} \\
\vdots \\
L_{m_{l o o p, n}}
\end{array}\right]=\left[\begin{array}{c}
\sum_{i=3}^{\infty} L_{m_{i, 1}} \\
\sum_{i=3}^{\infty} L_{m_{i, 2}} \\
\vdots \\
\sum_{i=3}^{\infty} L_{m_{i, n}}
\end{array}\right]
$$

By considering the rotor space harmonics, an emf is induced in one loop by the mmf in another loop. This is accounted for by considering the mutual inductance, $M_{x, y}$, for each loop $x$ in relation to another loop $y$. Noting that the self inductance of the principal fields (e.g $M_{x, x}$ ) are excluded. In calculating these mutual inductances, and indeed the magnetizing inductances, the effect of all the nests is included.

$$
[M]=\left(\frac{\mu_{0} l d q}{\pi g}\right)\left[\begin{array}{cccc}
0 & \sum_{i=3}^{\infty} \frac{k_{w_{1, i}} k_{w_{2, i}}}{\left(p_{i}\right)^{2}} & \ldots & \sum_{i=3}^{\infty} \frac{k_{w_{1, i}} k_{w_{n, i}}}{\left(p_{i}\right)^{2}} \\
\sum_{i=3}^{\infty} \frac{k_{w_{2, i}} k_{w_{1, i}}}{\left(p_{i}\right)^{2}} & 0 & \ldots & \sum_{i=3}^{\infty} \frac{k_{w_{2, i}} k_{w_{n, i}}}{\left(p_{i}\right)^{2}} \\
\vdots & \vdots & \ddots & \vdots \\
\sum_{i=3}^{\infty} \frac{k_{w_{n, i}} k_{w_{1, i}}}{\left(p_{i}\right)^{2}} & \sum_{i=3}^{\infty} \frac{k_{w_{n, i} k_{w_{2, i}}}}{\left(p_{i}\right)^{2}} & \ldots & 0
\end{array}\right]
$$

\section{B. Rotor-stator coupling via space harmonics}

Space harmonics produced by the rotor will also couple to the stator and effect rotor parameters. The stator resistance and reactance referred to the rotor (can be considered a short circuit on stator 1) is calculated as:

$$
\begin{gathered}
R_{s 1}^{\prime}=\frac{R_{s 1}\left(\frac{N_{e f f_{r}}}{N_{e f f_{s 1}}}\right)^{2}}{s_{1}} \\
X_{s 1}^{\prime}=j \omega L_{s 1}\left(\frac{N_{e f f_{r}}}{N_{\text {eff } f_{s 1}}}\right)^{2}
\end{gathered}
$$


Where $R_{s 1}$ and $L_{s 1}$ is the stator resistance and inductance respectively. $N_{e f f_{r}}$ and $N_{e f f_{s 1}}$ is the rotor and stator effective turns for the harmonic being analyzed and $s_{1}$ is the slip calculated in (11) extended to include all space harmonics. Similar equations can be applied to calculated $R_{s 2}^{\prime}$ and $X_{s 2}^{\prime}$ assuming a short circuit on stator 2 and using $s_{2}$ calculated in (12).

The total impedance, not including the effect of mutual couplings via space harmonics, is given as the sum of rotor coils resistance, $R_{n}$, the conventional leakage inductance components, overhang, slot and zig-zag, $\left(L_{r_{n}}\right)$ found using the methods described in [14] and the magnetizing inductance $\left(L_{m_{\text {coil }}}\right)$ in parallel with the referred stator impedance $\left(Z_{s}^{\prime}=R_{s}^{\prime}+X_{s}^{\prime}\right)$.

$$
[Z]=\operatorname{diag}\left(R_{n}+j \omega L r_{n}+\frac{\left(j \omega L_{m_{\text {coil }}}\right)\left(Z_{s}^{\prime}\right)}{j \omega L_{m_{\text {coil }}}+Z_{s}^{\prime}}\right)
$$

The current flowing through the individual rotor loops, for unit voltage applied to the 1 turn coil is given by:

$$
\left[\begin{array}{c}
I_{1} \\
I_{2} \\
\vdots \\
I_{n}
\end{array}\right]=([M]+[Z])^{-1}\left[\begin{array}{c}
k_{w_{1,1}} \\
k_{w_{1,2}} \\
\vdots \\
k_{w_{1, n}}
\end{array}\right]
$$

Using the current calculated in (25), the impedance, $Z_{\text {eff }}$, of the individual loops, $n$, in the nested loop rotor taking into account rotor-rotor and rotor-stator coupling via space harmonics is:

$$
\left[\begin{array}{c}
Z_{\text {eff }} \\
Z_{\text {ef } f_{2}} \\
\vdots \\
Z_{\text {eff }}
\end{array}\right]=\left[\begin{array}{c}
\left(\frac{k_{w_{1,1}}}{I_{1}}\right) \\
\left(\frac{k_{w_{1,2}}}{I_{2}}\right) \\
\vdots \\
\left(\frac{k_{w_{1, n}}}{I_{n}}\right)
\end{array}\right]
$$

\section{Parameter calculation}

The impedance presented by one loop, referred to the fictitious $p_{1}$ stator winding, can be determined by considering a short circuit on stator 2. The loop impedances are taken from (26). Multiplying this by $k_{w_{1 i}}{ }^{2}$ refers these to the one-turn stator winding and the impedance presented by one nest is given by the parallel combination of the loop impedances as shown in (27).

$$
Z_{e q}=\left(\sum_{i=1}^{n} \frac{k_{w_{1 i}}^{2}}{Z_{e f f_{i}}}\right)^{-1}
$$

A further step is the transformation from the $p_{1}+p_{2}$ phase system of the rotor to the three-phase system of the stator by multiplying by $\frac{p_{1}+p_{2}}{3}$; this point is discussed in [7]. It is important to note that these parameters are rotor frequency, i.e. speed, dependent.

The turns ratio for the nested loop rotor can be found by considering an mmf balance with one stator open circuit: [7]

$$
n_{r}=\sum_{i=1}^{n}\left|\frac{\left(\frac{k_{w_{1 i}} k_{w_{2 i}}}{Z_{i}}\right)}{\left(\frac{k_{w_{2 i}}{ }^{2}}{Z_{i}}\right)}\right|
$$

These rotor parameters are for the full equivalent circuit shown in figure $2 \mathrm{a}$.

\section{COMPARISON OF PARAMETER VALUES}

A comparison between the coupled-circuit and winding-factor method was carried out using two BDFMs, a frame size D180 machine [8] and a D400, $250 \mathrm{~kW}$ machine [3]. Stator parameters calculated from machine geometry are given in Table III. To account for the magnetic wedges fitted between the stator teeth of the D400 machine, a modified stator Carter factor was calculated using the procedure described in [6]. Rotor parameters found using the coupled-circuit and winding factor methods, evaluated at natural speed, are given in Table IV and are in close agreement. As discussed in the Section III, rotor parameters values obtained from the winding factor method are speed dependent, as shown in Table V. However, the variation with speed is negligible.

Table VI shows the effect on rotor parameters if:

(i) All space harmonic couplings: rotor-stator and rotor-rotor, generated by the rotor are included. 
TABLE III: Stator parameters calculated from machine geometry

\begin{tabular}{rcc}
\hline Parameter & $\mathrm{D} 180$ & $\mathrm{D} 400$ \\
\hline$R_{1}(\Omega)$ & 2.3 & 0.0907 \\
$R_{2}(\Omega)$ & 4 & 0.667 \\
$L_{1}(\mathrm{mH})$ & 5.34 & 2.73 \\
$L_{m_{1}}(\mathrm{mH})$ & 410 & 95.6 \\
$L_{2}(\mathrm{mH})$ & 14.8 & 45.9 \\
$L_{m_{2}}(\mathrm{mH})$ & 417 & 306 \\
\hline
\end{tabular}

TABLE IV: Comparison of coupled-circuit and winding factor methods for determining rotor parameters

\begin{tabular}{cccc}
\hline Machine & Parameter & Coupled-Circuit & Winding Factor \\
\hline \multirow{4}{*}{ D180 } & $R_{r}^{\prime}(\Omega)$ & 1.64 & 1.62 \\
& $L_{r}^{\prime}(\mathrm{mH})$ & 42.4 & 42.3 \\
& $n_{r}$ & 0.718 & 0.732 \\
\hline \multirow{4}{*}{ 400 } & $R_{r}^{\prime}(\Omega)$ & 0.109 & 0.109 \\
& $L_{r}^{\prime}(\mathrm{mH})$ & 6.09 & 6.28 \\
& $n_{r}$ & 0.401 & 0.409 \\
\hline
\end{tabular}

TABLE V: Rotor parameters found by the winding factor method as a function of speed

\begin{tabular}{ccccc}
\hline Speed & \multicolumn{2}{c}{$\mathrm{D} 180$} & \multicolumn{2}{c}{$\mathrm{D} 400$} \\
(rev/min) & $L_{r}^{\prime}(\mathrm{mH})$ & $R_{r}^{\prime}(\Omega)$ & $L_{r}^{\prime}(\mathrm{mH})$ & $R_{r}^{\prime}(\Omega)$ \\
\hline 350 & 42.37 & 1.621 & 6.275 & 0.10899 \\
500 & 42.30 & 1.623 & 6.275 & 0.10903 \\
650 & 42.26 & 1.627 & 6.275 & 0.10906 \\
\hline
\end{tabular}

(ii) Only rotor-rotor coupling via space harmonics are included.

(iii) No coupling via space harmonics included.

It can be seen that rotor-stator coupling has a very small effect on rotor parameters and in most cases does not need to be considered in contrast to rotor-rotor space harmonic coupling.

TABLE VI: Comparison of different winding factor methods for calculating rotor parameters at natural speed

\begin{tabular}{clccc}
\hline Machine & Parameter & All coupling & No R-S & No R-R \\
& & \\
\multirow{3}{*}{ D180 } & $R_{r}^{\prime}(\Omega)$ & 1.62 & 1.65 & 1.67 \\
& $L_{r}^{\prime}(\mathrm{mH})$ & 42.3 & 42.9 & 65.4 \\
& $n_{r}$ & 0.732 & 0.732 & 0.721 \\
\hline \multirow{3}{*}{ D400 } & $R_{r}^{\prime}(\Omega)$ & 0.109 & 0.109 & 0.111 \\
& $L_{r}^{\prime}(\mathrm{mH})$ & 6.28 & 6.30 & 10.48 \\
& $n_{r}$ & 0.409 & 0.409 & 0.408 \\
\hline
\end{tabular}

\footnotetext{
${ }^{1}$ No rotor - stator coupling via space harmonics

${ }^{2}$ No rotor - rotor, or rotor - stator coupling via space harmonics
}

\section{EXPERIMENTAL PARAMETER EXTRACTION}

Machine parameters were extracted from the D180 BDFM and $250 \mathrm{~kW}$ machine from cascade tests using a modification of the procedure described by Roberts et al. [8] in which parameters were obtained from experimentally determined torque-speed characteristics in the cascade mode. Finding the best fit to experimental data is done using nonlinear least squares optimization. To avoid being trapped in local minima, the Levenberg-Marquardt Algorithm (LMA) [15] has been employed instead of the 
simple random search used in [8]. The LMA shows better resilience against local minima, and converges faster and more reliably, especially by using multiple start points. In this case, a speed increase of about 100 times was achieved using 100 starting points.

Accuracy was improved in comparison to [8] by fixing parameters that can be accurately measured or to which the fitting shows little sensitivity. The stator winding resistances, $R_{1}$ and $R_{2}$, were obtained from DC measurements at working temperature (the skin effect can be ignored). The sum of $L_{1}$ and $L_{m_{1}}$, and $L_{2}$ and $L_{m_{2}}$ was determined from a no-load test applied, in turn, to each stator winding, with the other open circuit. The conditions under which the parameters were extracted are described in [16].

The D180 experimental and FEA results were obtained from cascade and induction mode tests carried out at a PW and CW voltage of $120 \mathrm{~V}, 50 \mathrm{~Hz}$. The D400 experimental and FEA results were carried out with the PW and CW supplied at $70 \mathrm{~V}$, $20 \mathrm{~Hz}$ and $240 \mathrm{~V}, 20 \mathrm{~Hz}$, respectively, due to restrictions on stator currents; hence, the extracted parameters do not represent the effect of iron saturation.

Experimentally extracted parameters are for the reduced form of the equivalent circuit, whereas the parameters obtained in the previous section are for the full model. Converting the full model to the reduced model using the method described in [8] enables a comparison to be made with the experimentally determined values and those found using the coupled-circuit and winding-factor methods. The results are shows in Table VII as well as the values found from FEA in [6]. The two analytical methods and FEA all lead to values of rotor resistance and turns ratio which are within $10 \%$ of the experimental values but the inductance parameters can show larger discrepancies. Inclusion of saturation effects (non-linear) in the FEA shows that the machines parameters are not greatly altered, showing that the iron circuits of these machines are conservatively designed.

With the D400 machine, there is about $15 \%$ difference in the rotor inductance, $L_{r}^{\prime}$. Abdi et al. [6] attributed this error to the difficulty in modelling leakage effects (e.g winding overhang). The agreement between FEA (linear) and FE (non-linear), both using identical geometrical data, is close, which shows that the non-linearity of the iron circuit can be neglected due to reduced voltage levels in the tests. Another reason for the discrepancy between analytical and experimental results is due to the variation of the air-gap along the length of the rotor as a consequence of aiming for a small air-gap which turned out to be a challenge to achieve. The air-gap variation will have an effect on the equivalent circuit parameters which assume a uniform air-gap.

TABLE VII: Reduced form of rotor and stator parameters extracted from coupled-circuit (CC), winding factor (WF), FEA and experimental (Exp) methods

\begin{tabular}{|c|c|c|c|c|c|c|}
\hline Machine & Parameter & $\mathrm{CC}$ & WF & FEA(linear) & FEA(non-linear) & Exp \\
\hline \multirow{7}{*}{ D180 } & $R_{r}^{\prime}(\Omega)$ & 1.68 & 1.67 & 1.53 & 1.53 & $\frac{1}{1.60}$ \\
\hline & $L_{r}^{\prime}(\mathrm{mH})$ & 56.5 & 56.7 & 56 & 56 & 54 \\
\hline & $n_{r}$ & 0.703 & 0.716 & 0.678 & 0.705 & 0.72 \\
\hline & $R_{1}(\Omega)$ & \multicolumn{4}{|c|}{2.30} & 2.42 \\
\hline & $R_{2}(\Omega)$ & \multicolumn{4}{|c|}{4.0} & 4.04 \\
\hline & $L_{m 1}(\mathrm{mH})$ & 415 & 416 & 425 & 431 & 457 \\
\hline & $L_{m 2}(\mathrm{mH})$ & 431 & 435 & 451 & 463 & 493 \\
\hline \multirow{7}{*}{ D400 } & $R_{r}^{\prime}(\Omega)$ & 0.115 & 0.115 & 0.120 & 0.122 & 0.114 \\
\hline & $L_{r}^{\prime}(\mathrm{mH})$ & 16.0 & 16.5 & 15.2 & 14.3 & 12.5 \\
\hline & $n_{r}$ & 0.359 & 0.366 & 0.355 & 0.365 & 0.380 \\
\hline & $R_{1}(\Omega)$ & \multicolumn{4}{|c|}{0.0907} & 0.0971 \\
\hline & $R_{2}(\Omega)$ & \multicolumn{4}{|c|}{0.667} & 0.706 \\
\hline & $L_{m 1}(\mathrm{mH})$ & 98.3 & 98.3 & 96.8 & 97.1 & 104 \\
\hline & $L_{m 2}(\mathrm{mH})$ & 352 & 383 & 349 & 341 & 368 \\
\hline
\end{tabular}

The experimental results, with all parameters referred to stator 1, are expressed in per-unit and shown in Table VIII to provide more generality to the results and help with comparisons between the two machines. As expected the per unit resistance is lower for the larger machines.

\section{CONCLUSIONS}

Two analytical procedures for obtaining rotor parameters for BDFMs with nested loop rotors have been described. One transforms a reduced coupled circuit model into a complex form to give speed independent values. An alternative procedure uses winding factors to give parameters valid for one operating condition but in fact there is little dependence on speed. Parameters determined by the two methods are close to those determined experimentally and by FEA. The ability to calculate parameters accurately enables the exploration of rotor designs, and the overall machine design. Although this study has looked at the nested loop type of rotor, the methods can be readily extended to other arrangements, including loops of arbitrary pitch 
TABLE VIII: Experiential results expressed in per-unit

\begin{tabular}{ccc}
\hline Parameter & D180 & D400 \\
\hline$R_{r}^{\prime}$ & 0.19 & 0.06 \\
$L_{r}^{\prime}$ & 2.06 & 2.06 \\
$R_{1}$ & 0.29 & 0.05 \\
$R_{2}^{\prime \prime}$ & 0.25 & 0.05 \\
$L_{m 1}$ & 17.45 & 17.16 \\
$L_{m 2}^{\prime \prime}$ & 9.76 & 8.77 \\
\hline
\end{tabular}

and placement. Using both analytical methods give a valuable cross-check, and the winding factor method gives more obvious insight into the effects of space harmonics. FEA, of course, is essential in assessing the effects of saturation.

\section{APPENDIX A}

EXPANSION OF COMPLEX SEQUENCE TERMS

$$
\omega_{r} Q_{c}^{+}+j \omega_{\text {sync }} M_{c}^{+}=\left[\begin{array}{cc|c}
j\left(L_{1}-M_{1}\right)\left(\omega_{\mathrm{sync}}-\omega_{r} p_{1}\right) & 0 & j\left(\omega_{\mathrm{sync}}-\omega_{r} p_{1}\right) M_{c_{s 1 r}} \\
0 & j\left(L_{2}-M_{2}\right)\left(\omega_{\mathrm{sync}}+\omega_{r} p_{2}\right) & j\left(\omega_{\mathrm{sync}}+\omega_{r} p_{2}\right) M_{c_{s 2 r}} \\
\hline j \omega_{\mathrm{sync}} M_{c_{s 1 r}}^{*} & j \omega_{\mathrm{sync}} M_{c_{s 2 r}}^{*} & j \omega_{\mathrm{sync}} M_{c_{r}}
\end{array}\right]
$$

The terms described in the complex sequence component derivation are expanded here:

$$
\begin{aligned}
& Q_{c}=\mathcal{T}_{\text {cmplx }} Q_{d q} \mathcal{T}_{\text {cmplx }}^{*}=\left[\begin{array}{ccc}
Q_{c}^{+} & 0 & 0 \\
0 & \bar{Q}_{c}^{+} & 0 \\
0 & 0 & 0
\end{array}\right] \\
& Q_{c}^{+}=\left[\left[\begin{array}{cc}
-j p_{1}\left(L_{1}-M_{1}\right) & 0 \\
0 & j p_{2}\left(L_{2}-M_{2}\right)
\end{array}\right] Q_{c_{s r}}^{+}\right] \\
& M_{c}=\mathcal{T}_{\text {cmplx }} M_{d q} \mathcal{T}_{\text {cmplx }}^{*}=\left[\begin{array}{ccc}
M_{c}^{+} & 0 & 0 \\
0 & \bar{M}_{c}^{+} & 0 \\
0 & 0 & M_{d q \emptyset}
\end{array}\right] \\
& R_{c}=\mathcal{T}_{\text {cmplx }} R_{d q} \mathcal{T}_{\text {cmplx }}^{*}=\left[\begin{array}{ccc}
R_{c}^{+} & 0 & 0 \\
0 & \bar{R}_{c}^{+} & 0 \\
0 & 0 & R_{d q \emptyset}
\end{array}\right]
\end{aligned}
$$

where $Q_{c}, M_{c}, R_{c} \in \mathbb{C}^{5 \times 5}, Q_{c}^{+}, M_{c}^{+}, R_{c}^{+} \in \mathbb{C}^{3 \times 3}, Q_{c_{s r}}^{+} \in \mathbb{C}^{2 \times 1}$, and:

$$
\begin{gathered}
Q_{c_{s r}}^{+}=\left[\begin{array}{c}
-j p_{1} M_{1 s r_{d q}} e^{j \phi_{1}} \\
j p_{2} M_{2 s r_{d q}} e^{j \phi_{2}}
\end{array}\right] \\
M_{c}^{+}=\left[\begin{array}{cc}
{\left[\begin{array}{cc}
L_{1}-M_{1} & 0 \\
0 & L_{2}-M_{2}
\end{array}\right]} & M_{c_{s r}}^{+} \\
M_{c_{s r}}^{+}{ }^{*} & M_{c_{r}}^{+}
\end{array}\right] \\
M_{c_{s r}}^{+}=\left[\begin{array}{l}
M_{1 s r_{d q}} e^{j \phi_{1}} \\
M_{2 s r_{d q}} e^{j \phi_{2}}
\end{array}\right] \\
M_{c_{r}}^{+}=L_{d q_{r}}
\end{gathered}
$$




\section{REFERENCES}

[1] R. Carlson, H. Voltolini, F. Runcos, P. Kuo-Peng, and N. Batistela, "Performance analysis with power factor compensation of a 75 kw brushless doubly fed induction generator prototype," in Electric Machines Drives Conference, 2007. IEMDC '07. IEEE International, vol. 2, May 2007, pp. 1502-1507.

[2] H. Liu and L. Xu, "Design and performance analysis of a doubly excited brushless machine for wind power generator application," in Power Electronics for Distributed Generation Systems (PEDG), 2010 2nd IEEE International Symposium on, June 2010, pp. 597-601.

[3] E. Abdi, R. McMahon, P. Malliband, S. Shao, E. Mathekga, P. Tavner, S. Abdi, A. Oraee, T. Long, and M. Tatlow, "Performance analysis and testing of a $250 \mathrm{kw}$ medium-speed brushless doubly-fed induction generator," Renewable Power Generation, IET, vol. 7, pp. 631 - 638, 2013.

[4] S. Williamson, A. C. Ferreira, and A. K. Wallace, "Generalised theory of the brushless doubly-fed machine. Part 1: Analysis," IEE Proceedings - Electric Power Applications, vol. 144, no. 2, pp. 111-122, 1997.

[5] P. Roberts, T. Long, R. McMahon, S. Shao, E. Abdi, and J. Maciejowski, "Dynamic modelling of the brushless doubly fed machine," Electric Power Applications, IET, vol. 7, no. 7, pp. 544-556, Aug 2013.

[6] S. Abdi, E. Abdi, A. Oraee, and R. McMahon, "Equivalent circuit parameters for large brushless doubly fed machines (bdfms)," Energy Conversion, IEEE Transactions on, vol. 29, no. 3, pp. 706-715, Sept 2014.

[7] R. McMahon, P. Tavner, E. Abdi, P. Malliband, and D. Barker, "Characterising brushless doubly fed machine rotors," Electric Power Applications, IET, vol. 7, no. 7, pp. 535-543, Aug 2013.

[8] P. C. Roberts, R. A. McMahon, P. J. Tavner, J. M. Maciejowski, and T. J. Flack, "Equivalent circuit for the brushless doubly-fed machine (BDFM) including parameter estimation," Proc. IEE B - Elec. Power App., vol. 152, no. 4, pp. 933-942, July 2005.

[9] R. Li, R. Spée, A. K. Wallace, and G. C. Alexander, "Synchronous drive performance of brushless doubly-fed motors," IEEE Transactions on Industry Applications, vol. 30, no. 4, pp. 963-970, July/August 1994

[10] R. A. McMahon, P. C. Roberts, X. Wang, and P. J. Tavner, "Performance of the BDFM as a generator and a motor," Proc. IEE B - Elec. Power App., March 2006

[11] B. V. Gorti, G. C. Alexander, and R. Spée, "Power balance considerations for brushless doubly-fed machines," IEEE Transactions on Energy Conversion, vol. 11, no. 4, pp. 687-692, December 1996.

[12] A. B. Carlson, Circuits: Engineering Concepts and Analysis of Linear Electric Circuits. John Wiley \& Sons Inc., New York, 1996.

[13] G. Slemon and A. Straughen, Electrical Machines. Addison-Wesley, 1980.

[14] A. Draper, Electrical Machines, 2nd ed. Longmans, 1967.

[15] J. C. Nash, Compact Numerical Methods for Computers: Linear Algebra and Function Minimisation, 2nd ed. Bristol: Adam Hilger, 1990.

[16] X. Wang, P. Roberts, and R. McMahon, "Optimisation of bdfm stator design using an equivalent circuit model and a search method," in Power Electronics, Machines and Drives, 2006. The 3rd IET International Conference on, April 2006, pp. 606-610. 